\title{
Functional Organization of the Parahippocampal Cortex: Dissociable Roles for Context Representations and the Perception of Visual Scenes
}

\author{
Oliver Baumann ${ }^{1}$ and ${ }^{-}$Jason B. Mattingley ${ }^{1,2}$ \\ ${ }^{1}$ Queensland Brain Institute and ${ }^{2}$ School of Psychology, The University of Queensland, St Lucia 4072, Australia
}

The human parahippocampal cortex has been ascribed central roles in both visuospatial and mnemonic processes. More specifically, evidence suggests that the parahippocampal cortex subserves both the perceptual analysis of scene layouts as well as the retrieval of associative contextual memories. It remains unclear, however, whether these two functional roles can be dissociated within the parahippocampal cortex anatomically. Here, we provide evidence for a dissociation between neural activation patterns associated with visuospatial analysis of scenes and contextual mnemonic processing along the parahippocampal longitudinal axis. We used fMRI to measure parahippocampal responses while participants engaged in a task that required them to judge the contextual relatedness of scene and object pairs, which were presented either as words or pictures. Results from combined factorial and conjunction analyses indicated that the posterior section of parahippocampal cortex is driven predominantly by judgments associated with pictorial scene analysis, whereas its anterior section is more active during contextual judgments regardless of stimulus category (scenes vs objects) or modality (word vs picture). Activation maxima associated with visuospatial and mnemonic processes were spatially segregated, providing support for the existence of functionally distinct subregions along the parahippocampal longitudinal axis and suggesting that, in humans, the parahippocampal cortex serves as a functional interface between perception and memory systems.

Key words: context; fMRI; parahippocampal cortex; parahippocampal gyrus; scene perception

Significance Statement

The functional neuroanatomy of the parahippocampal cortex is still subject to considerable debate. Specifically, its relative contributions to visuospatial and mnemonic functions remain unclear. This study constitutes the first evidence for the existence of distinct information-processing properties along the parahippocampal longitudinal axis. Our findings implicate the posterior section of the parahippocampus in visuospatial perception and the anterior section in contextual mnemonic processes. Our study provides novel neuroanatomical information critical for understanding the diversity of the purported functions of the human parahippocampal cortex.

\section{Introduction}

Various lines of evidence indicate that the human parahippocampal cortex plays a key role in both visuospatial (Epstein et al., 1999, 2003; Park and Chun, 2009; Rajimehr et al., 2011) and

\footnotetext{
Received Sept. 9, 2015; revised Jan. 19, 2016; accepted Jan. 25, 2016.

Author contributions: 0. B. and J.B.M. designed research; 0 .B. performed research; $0 . B$ analyzed data; $0 . B$. and J.B.M. wrote the paper.

This work was supported by the Australian Research Council (ARC) (Discovery Early Career Researcher Award DE120100535 to 0.B; ARC Australian Laureate Fellowship FL110100103 to J.B.M.), the ARC-SRI Science of Learning Research Centre (Grant SR120300015), and the ARC Centre of Excellence for Integrative Brain Function (ARC Centre Grant CE140100007)

The authors declare no competing financial interests.

Correspondence should be addressed to Oliver Baumann, Queensland Brain Institute, the University of Queensland, St Lucia, Queensland, 4072, Australia. E-mail: o.baumann@uq.edu.au.

DOI:10.1523/JNEUROSCI.3368-15.2016

Copyright $\odot 2016$ the authors $\quad 0270-6474 / 16 / 362536-07 \$ 15.00 / 0$
}

mnemonic (Bar and Aminoff, 2003; Düzel et al., 2003; Janzen and van Turennout, 2004; Baumann et al., 2010; Wegman and Janzen, 2011) processes. Evidence for a role of the parahippocampal gyrus in visuospatial analysis derives mainly from human neuroimaging experiments, which have shown that this region responds strongly to visual scenes such as landscapes, cityscapes, and buildings (giving rise to the name parahippocampal place area, PPA; Epstein and Kanwisher, 1998; Epstein, 2005; Epstein and Ward, 2010). Increased activity within the posterior aspect of the parahippocampal cortex during viewing of scenes relative to objects has been suggested to reflect regional specialization for visuospatial processes related to the extraction of scene layout information (Epstein, 2008). The idea that the posterior parahippocampal cortex serves a dedicated role in visuospatial processing is further supported by studies showing that this brain region 
is sensitive to changes in basic visuospatial properties such as the shape and spatial frequency of abstract geometric objects and layouts (Rajimehr et al., 2011; Zeidman et al., 2012).

In addition to its role in visuospatial analysis, the parahippocampal cortex has also been implicated in the integration and retrieval of contextual associations in memory (Aminoff et al., 2013; Bar and Aminoff, 2003; Bar et al., 2008a, 2008b). Contextual associations are defined as relations between objects and conditions that describe, represent, and bring meaning to an environment (Aminoff et al., 2013). Examples of such associations are objects that commonly co-occur, such as a refrigerator and an oven (typically encountered in a kitchen) or familiar spatial configurations of objects, such as rows of tables and chairs in a school classroom. According to this view, activity in the parahippocampal cortex reflects modality-independent retrieval of contextual information from memory, such as which objects typically occur in a given scene and where they are likely to be located relative to one another.

Although there is evidence that the parahippocampal cortex is involved in the visuospatial analysis of scenes as well as contextual processing, it remains unknown whether these two functions can be dissociated anatomically. A recent fMRI study (Baldassano et al., 2013) reported that anterior voxels in the parahippocampal cortex are more strongly functionally connected to parietal and medial temporal networks, whereas posterior voxels are more strongly functionally connected to occipital regions. This anatomical segregation of function suggests a predominantly perceptual role for the posterior parahippocampal cortex and a mnemonic role for the anterior parahippocampal cortex. (Note that the anterior parahippocampal cortex should not be confused with the anterior portion of the parahippocampal gyrus, which includes the perirhinal cortex; Ranganath and Ritchey, 2012.) Here, we tested this hypothesis directly by determining whether neural activity associated with visuospatial analysis of scenes can be functionally dissociated from activity associated with the processing of mnemonic contextual information.

We used fMRI to measure parahippocampal responses while participants engaged in a task that required them to judge the contextual relatedness of scene and object pairs (e.g., "bike" and "bell" are a contextually congruent object-object pair, whereas "sofa" and "microscope" are incongruent; "boat" and "lake" are a contextually congruent object-scene pair, whereas "tractor" and "hotel" are incongruent). Each pair was presented either in printed-word format or in a combined word-picture format, allowing us to present pictorial objects and scenes in isolation (Fig. 1 ). To identify visuospatial regions of the parahippocampal cortex, we tested for voxels with greater activity for picture-scene trials than for picture-object trials over and above activity differences elicited by the comparison of word-scene trials and wordobject trials. In addition, to locate context-related regions of the parahippocampal cortex, we tested for voxels that were selectively active during the contextual judgment task regardless of the type of stimulus (scenes vs objects) or presentation modality (word vs picture).

\section{Materials and Methods}

Participants. Twenty-four healthy, adult volunteers gave their informed consent to participate in the study, which was approved by the Human Research Ethics Committee of The University of Queensland. The participants (12 females) ranged in age from 18 to 33 years (mean, 21 years) and all were right-handed. All participants were fluent English readers.

Stimuli and procedure. Each trial consisted of a $2 \mathrm{~s}$ presentation of an object-object pair or object-scene pair presented either in printed-word
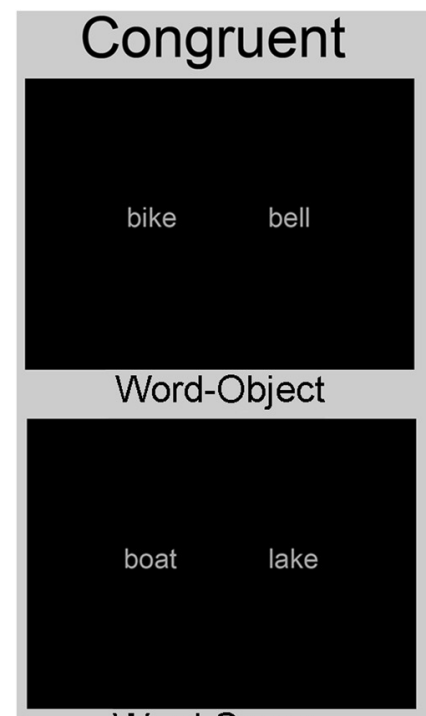

Word-Scene

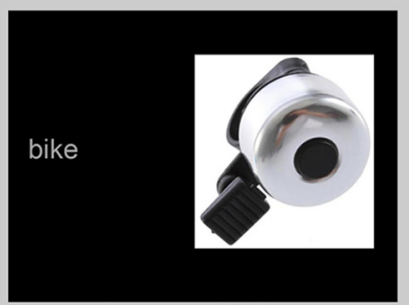

Picture-Object

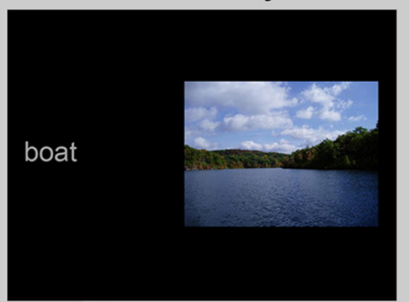

Picture-Scene

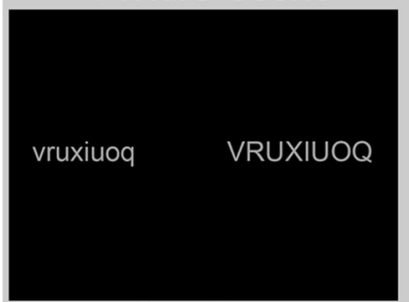

Baseline
Incongruent

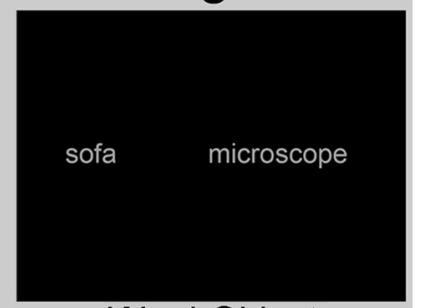

Word-Object

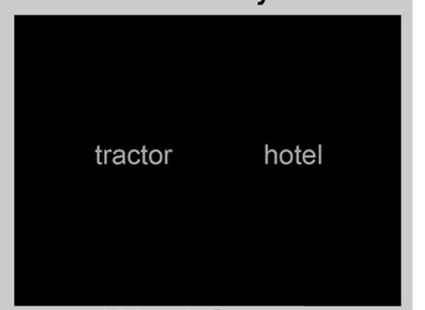

Word-Scene

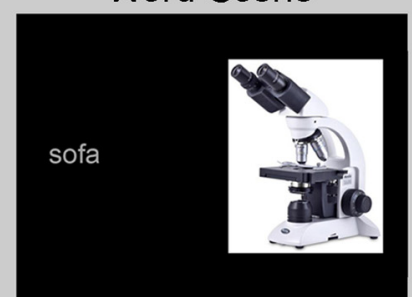

Picture-Object

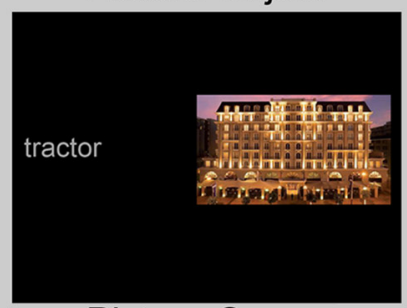

Picture-Scene

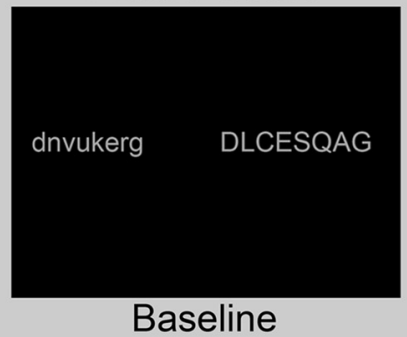

Figure 1. Examples of stimulus pairs from the ten experimental conditions. The participants' task was to judge whether a given stimulus pair was contextually congruent or incongruent (e.g., "boat" and "lake" are contextually congruent, whereas "tractor" and "hotel" are incongruent). The baseline conditions required participants to judge whether two random-letter strings (one uppercase and one lowercase) were the same or different.

format or in a combined word-picture format (Fig. 1). This design allowed us to present pictorial objects and scenes in isolation and to contrast their visually evoked activity patterns. The participants' task was to judge whether a stimulus pair was contextually congruent or incongruent (e.g., "boat" and "lake" are contextually congruent, whereas "tractor" and "hotel" are not) and to indicate their decision via button press within $4 \mathrm{~s}$ of stimulus onset. The response period was followed by a variable blank interval of $0-2 \mathrm{~s}$. In addition to the verbal and pictorial task conditions, we used an active baseline condition that required participants to compare two random-letter strings (one uppercase and one lowercase) and determine whether they were the same or different (Fig. 1). This 
condition was comparable in visual and response demands to the verbal condition, but did not involve contextual retrieval processes (i.e., access to semantic information), thus providing a baseline to test for a conjoint effect of contextual processing across the other eight task conditions.

Each object-object and object-scene pair was presented only once to each participant, either as printed words or pictorially, to avoid learning effects. To control for the effects of individual stimuli, object-object and object-scene pairs that were presented to half of the participants as words were presented to the other participants as pictures and vice versa. Thirty trials were presented for each of the ten task conditions. The resulting 300 trials were divided into 4 imaging runs lasting $\sim 6.5 \mathrm{~min}$ each.

Stimuli were color photographs of everyday objects and scenes (425 pixels in their longest dimension) and letter strings (number of characters, 3-17; font, Arial; font size, 45). Stimuli were presented either in printed-word format or in a combined word-picture format (with pictures always on the right side) using Presentation software (Neurobehavioral Systems; http://www.neurobs.com) running on a PC connected to a liquid crystal display projector $(1024 \times 768$ resolution $)$ that back projected stimuli onto a screen positioned at the head end of the scanner bed. Participants lay on their back within the bore of the magnet and viewed the stimuli via a mirror that reflected the images displayed on the screen. The distance to the screen was $90 \mathrm{~cm}$ ( $12 \mathrm{~cm}$ from eyes to mirror) and the visible part of the screen encompassed $\sim 22.0^{\circ} \times 16.4^{\circ}$ of visual angle $(35.5 \times 26 \mathrm{~cm})$.

MRI acquisition. Brain images were acquired on a $3 \mathrm{~T}$ MR scanner (Trio; Siemens) fitted with a 12-channel head coil. For the functional data, 29 axial slices (slice thickness, $3 \mathrm{~mm}$ ) were acquired in a descending order using a gradient echo echoplanar $\mathrm{T} 2{ }^{\star}$-sensitive sequence (repetition time, $1.8 \mathrm{~s}$; echo time, $30 \mathrm{~ms}$; flip angle, $80^{\circ}$; matrix, $64 \times 64$; field of view, $192 \times 192 \mathrm{~mm}$; in-plane resolution, $3 \times 3 \mathrm{~mm}$; phase encoding polarity, positive). In each of four runs, 219 volumes were acquired for each participant; the first four images were discarded to allow for T1 equilibration. The sequence was optimized to minimize signal dropouts in the medial temporal lobes (Weiskopf et al., 2006) and geometric distortions in the EPI images caused by magnetic field inhomogeneities were corrected using a point-spread mapping approach (Zeng and Constable, 2002; Zaitsev et al., 2003). We also acquired a T1-weighted structural MPRAGE scan. To minimize head movement, all participants were stabilized with tightly packed foam padding surrounding the head.

Data analysis. Image processing and statistical analyses were performed using SPM12 (Wellcome Department of Imaging Neuroscience, University College London). Functional data volumes were slice-time corrected and realigned to the first volume. A T2*-weighted mean image of the unsmoothed images was coregistered with the corresponding anatomical T1-weighted image from the same individual. The individual T1 image was used to derive the transformation parameters for the stereotaxic space using the SPM12 template (Montreal Neurological Institute template), which was then applied to the individual coregistered EPI images. Given our hypothesis-driven approach and to maximize the sensitivity of our fMRI analyses, we used region of interest (ROI) analyses focused on the left and right parahippocampal gyrus. ROIs were defined using the AAL atlas (Tzourio-Mazoyer et al., 2002) and WFU pickatlas tool (Maldjian et al., 2003).

In the first-level analysis, we generated a model incorporating 10 experimental task regressors (baseline-same, baseline-different, wordobject-congruent, word-object-incongruent, word-scene-congruent, word-scene-incongruent, picture-object-congruent, picture-objectincongruent, picture-scene-congruent, and picture-scene-incongruent; Fig. 1a). In the second-level analysis, an ROI-based SPM full factorial ANOVA with factors of stimulus category (object-object, object-scene) and modality (word, picture) was conducted. To identify parahippocampal regions that were predominantly engaged during visuospatial scene analysis (i.e., regions that showed greater activity for picture-scene trials than picture-object trials over and above activity differences elicited by the comparison of word-scene trials and word-object trials), we tested whether any voxels within the parahippocampal gyrus showed a significant interaction of the factors modality and stimulus category (threshold, $p \leq 0.05$, FWE corrected at the voxel level, extend threshold, 10), followed by post hoc extraction of parameter estimates of regional peak activity. Finally, to identify regions of the parahippocampal gyrus that were significantly engaged across all contextual judgment tasks (i.e., activity common to all tasks), we performed a conjunction analysis (using a conjunction-null hypothesis) across these task conditions relative to the random-letter baseline conditions (Nichols et al., 2005; threshold, $p \leq 0.05$ FWE corrected at the voxel-level, extent threshold, 10). The role of a baseline condition in a conjunction analysis is to remove common activity of no interest, which in this case is activity related to general attentional and response-related processes.

\section{Results}

\section{Behavioral performance}

For the categorization of object-object and object-scene pairs as either contextually congruent or incongruent, there was a remarkable degree of consistency across the participants' judgments. In the eight contextual judgment task conditions, participants made, on average, only $2.27 \%$ incorrect judgments. Errors of omission were even rarer $(0.17 \%)$. It took participants, on average, $1.41 \mathrm{~s}(\mathrm{SE}=0.04 \mathrm{~s})$ to respond in the eight contextual judgment task conditions. We compared response times across all conditions (paired $t$ test, threshold, $p \leq 0.05$ ) and found no significant difference between them, with the exception of the picture-object conditions, in which participants responded, on average, $0.09 \mathrm{~s}$ faster than in the other conditions.

\section{fMRI results}

\section{Parahippocampal gyrus}

To identify subregions of the parahippocampal cortex associated with picture-scene processing, we conducted a two-step statistical analysis. First, we used a factorial analysis to identify voxels that displayed a significant interaction between the factors of stimulus category (objects or scenes) and modality (pictures or words). This analysis identified two activity clusters located in the posterior section of the left (peak voxel $=-28,-44,-6$, cluster size $=97$ voxels, $F=94.03$ ) and right parahippocampal cortex (peak voxel $=28,-42,-8$, cluster size $=189$ voxels, $F=183.58$; Fig. $2 a$ ). In a second step, we extracted the parameter estimates from the two activation maxima yielded by the factorial analysis, separately for congruent and incongruent trials, to identify which condition was driving the interaction. As can be seen in Figure $2 b$, the interaction was clearly driven by stronger activity in the picture-scene conditions relative to the other task conditions (Fig. $2 b$ ). The parahippocampal response was significantly stronger bilaterally for object-scene than object-object pairs in the pictorial conditions, but not in the word conditions (two-tailed dependent $t$ test, threshold, $p \leq 0.05$ ). There was no significant effect of congruency (congruent vs incongruent trials; repeatedmeasures ANOVA, threshold, $p \leq 0.05$; Fig. 2b). This suggests that the posterior section of the parahippocampal cortex underlies perception-based scene analysis, but not more general contextual mnemonic processing. In addition, voxelwise analysis of the parahippocampal ROIs showed that activity in the word conditions was not significantly different from activity in the baseline conditions. Together, these findings suggest that a posterior section of the parahippocampal cortex underlies perception-based scene analysis, but not more general contextual mnemonic processing.

To identify any subregions of the parahippocampal cortex engaged by contextual mnemonic processes, regardless of stimulus category (objects or scenes) or modality (pictures or words), we conducted a conjunction analysis, testing for common activity across all contextual judgment task conditions relative to baseline. This analysis revealed a single left-hemisphere cluster located in the anterior parahippocampal cortex (peak voxel $=-28$, 


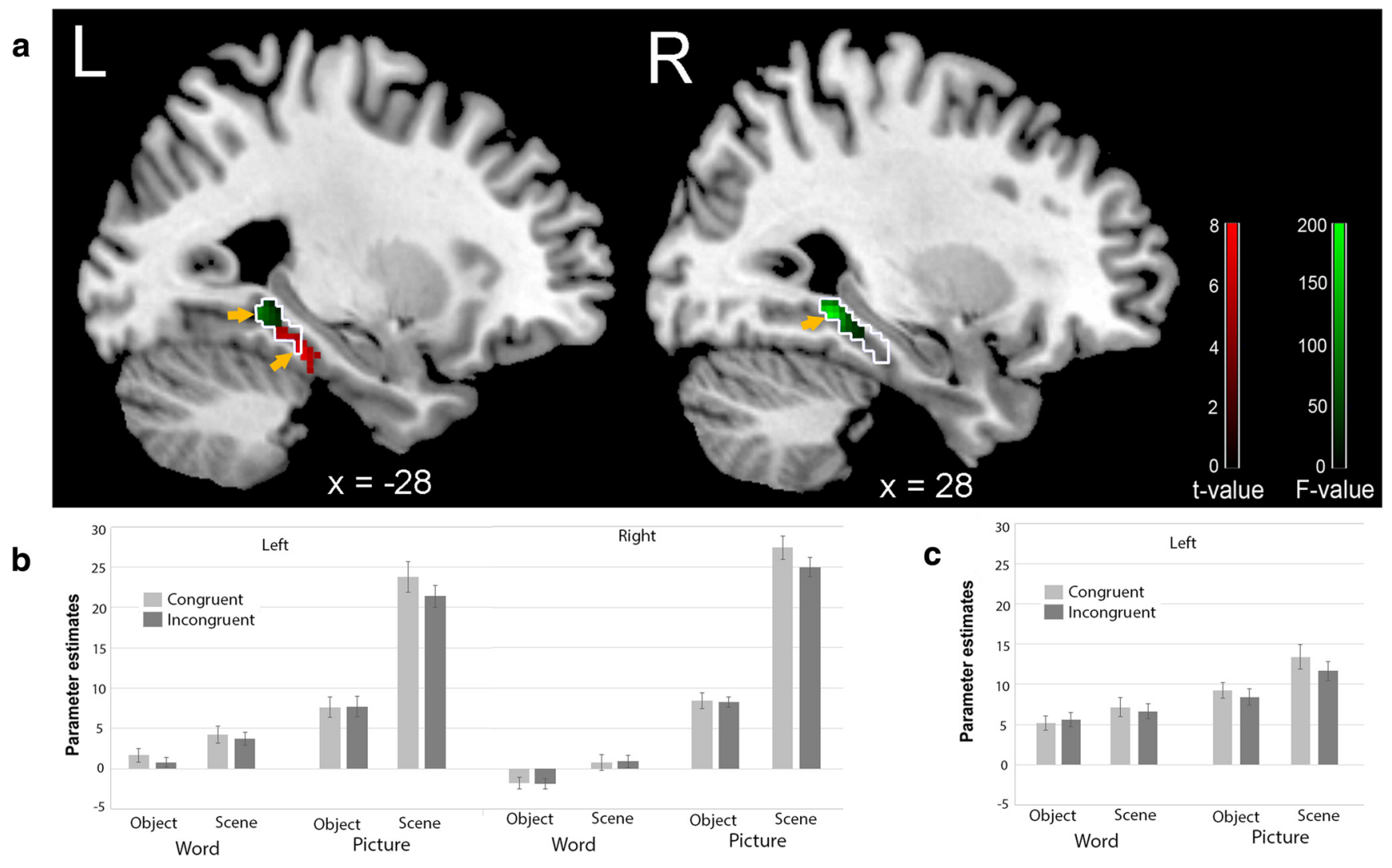

Figure 2. Results for the parahippocampal ROI analyses. $\boldsymbol{a}$, Sagittal MRI brain slices depicting results from the interaction analysis (green) and conjunction analysis (red); yellow arrows indicate activation maxima. The area outlined in white shows the PPA inferred from the contrast picture-scene $>$ picture-object. $\boldsymbol{b}$, Parameter estimates ( \pm 1 SE) from the interaction analysis shown separately for the eight contextual judgment task conditions (relative to baseline) and for left and right parahippocampal cortex. $c$, Parameter estimates ( \pm 1 SE) from the conjunction analysis shown separately for the eight contextual judgment task conditions (relative to baseline) for the left parahippocampal cortex.

$-32,-18$, cluster size $=58$ voxels, $t=8.01$; Fig. $2 a, c)$. This result suggests that a more anterior section of the left parahippocampal cortex underlies contextual mnemonic processing regardless of the visual properties (picture vs word) and stimulus category (objects or scenes) of the stimulus material. As shown in Figure $2 a$, the activation maxima associated with visuospatial and mnemonic processes were spatially well separated and there was virtually no overlap between the activation clusters, providing further support for the existence of functionally distinct subregions in the posterior and anterior parahippocampal cortex. To further statistically investigate the functional dissociation between these two putative subregions, we extracted and compared the peak voxel parameter estimates of the interaction and conjunction analyses separately for the anterior $(-28,-32,-18)$ and posterior $(-28,-44,-6)$ parahippocampal ROIs. The interaction effect was significantly stronger in the posterior ROI (average parameter estimate $=24.36, \mathrm{SEM}=2.51$ ) than in the anterior ROI (average parameter estimate $=4.76, \mathrm{SEM}=1.66$; independent $t$ test, $t=6.51, \mathrm{p}=<0.0001)$. In contrast, the conjunction effect was significantly stronger in the anterior ROI (average parameter estimate $=12.11, \mathrm{SEM}=1.51$ ) than in the posterior ROI (average parameter estimate $=2.46$, $\mathrm{SEM}=2.29$; independent $t$ test, $t=3.52, p<0.001$ ). It is important to note that we have shown peak-voxel parameter estimates here to highlight the maximal differences in response characteristics of the two subregions. We note that these differences are less pronounced for voxels at the borders between the anterior and posterior clusters and that the precise location and extent of anterior and posterior subregions is likely to vary between individual par- ticipants. Finally, it should be noted that, as is the case with all voxelwise fMRI investigations, the fact that voxels within the anterior and posterior parahippocampal cortex are significantly activated by different fMRI contrasts does not logically demonstrate a functional double dissociation between these two regions.

To assess the relative location of the two identified parahippocampal subregions relative to the location of the PPA (Epstein and Kanwisher, 1998), we used the contrast picture-scene $>$ picture-object to approximate its location (see white outline in Fig. 2a). It is evident that the posterior scene-sensitive subregions of the parahippocampal cortex fall entirely within the PPA, whereas the anterior context-related parahippocampal cortex falls only partially within the PPA. It should be noted, however, that the picture-scene $>$ picture-object contrast can only serve as an approximation for the PPA because it was not independently derived. In addition, given that our experimental task involved contextual judgments, the contrast of picture-scene $>$ pictureobject is likely to have encapsulated not only visuospatial influences, but also contextual differences between scenes and objects.

\section{Whole brain}

To put our results into the context of more widespread visual and memory networks, we also performed exploratory whole-brain analyses. Using the factorial analysis described in the previous section, we identified several additional clusters that displayed a significant interaction between the factors of stimulus category (objects or scenes) and modality (pictures or words). The highest level of activity was observed in a bilateral cluster encompassing 


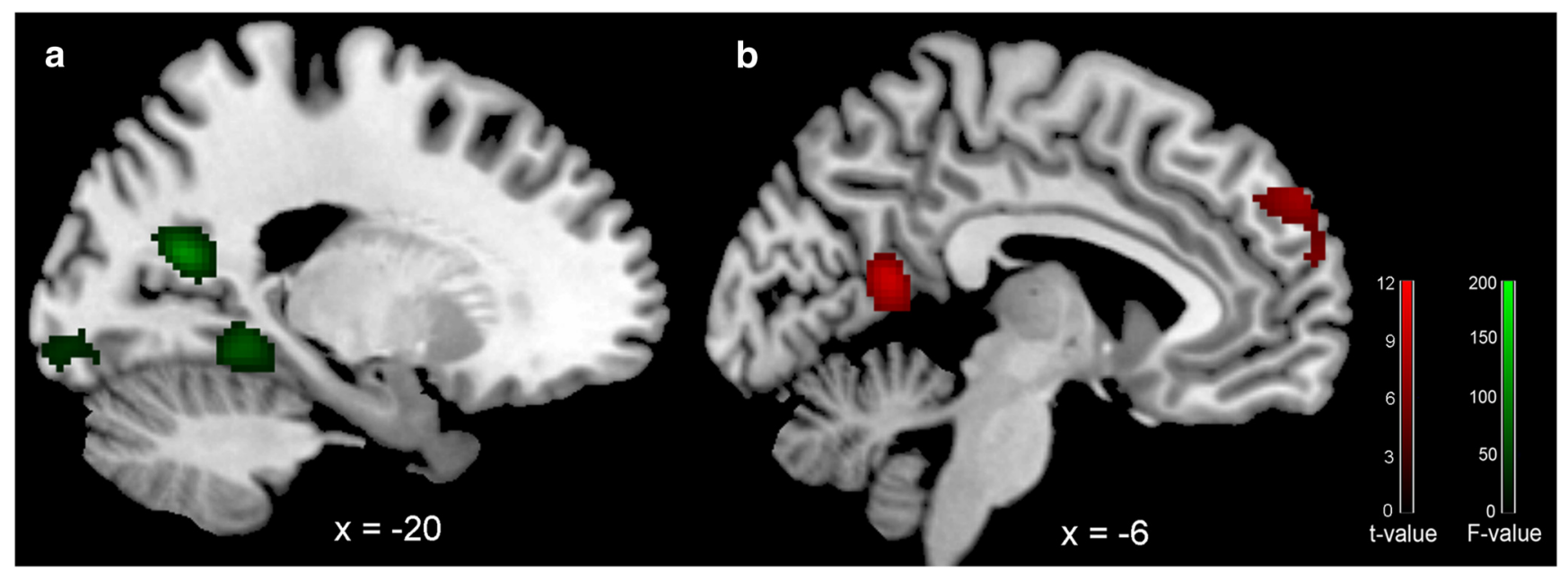

Figure 3. Results from the whole-brain analyses. $\boldsymbol{a}$, Sagittal MRI brain slices depicting results from the interaction analysis (green). $\boldsymbol{b}$, Sagittal MRI brain slices depicting results from the conjunction analysis (red).

Table 1. Summary of fMRI findings for the interaction analysis

\begin{tabular}{|c|c|c|c|c|c|c|c|}
\hline \multirow[b]{2}{*}{ Region } & \multirow[b]{2}{*}{ Hemisphere } & \multirow{2}{*}{$\begin{array}{l}\text { Brodmann } \\
\text { area }\end{array}$} & \multicolumn{3}{|c|}{$\begin{array}{l}\text { Activation maxima } \\
\text { MNI coordinates }\end{array}$} & \multirow{2}{*}{$\begin{array}{l}F \text {-values/ } \\
Z \text {-values of } \\
\text { maxima }\end{array}$} & \multirow{2}{*}{$\begin{array}{l}\text { Cluster } \\
\text { size (no. } \\
\text { of voxels }\end{array}$} \\
\hline & & & $x$ & $y$ & $z$ & & \\
\hline PHC & $\mathrm{R}$ & $30 / 37$ & 28 & -42 & -8 & 183.58/Inf & 557 \\
\hline $\begin{array}{l}\text { Retrosplenial } \\
\text { cortex }\end{array}$ & L & $23 / 29 / 30$ & -20 & -62 & 18 & $110.22 /$ Inf & 276 \\
\hline PHC & L & $30 / 37$ & -26 & -48 & -6 & 109.60/Inf & 468 \\
\hline $\begin{array}{l}\text { Retrosplenial } \\
\text { cortex }\end{array}$ & R & $23 / 29 / 30$ & 20 & -52 & 14 & $107.10 / \operatorname{lnf}$ & 541 \\
\hline Calcarine & $\mathrm{R}$ & 18 & 18 & -90 & -2 & 71.05/7.14 & 388 \\
\hline Calcarine & L & 18 & -12 & -92 & -8 & $65.07 / 6.90$ & 309 \\
\hline Cuneus & $L / R$ & 17/18/19 & -6 & -82 & 22 & $62.52 / 6.79$ & 1614 \\
\hline MOG & $\mathrm{R}$ & 19 & 40 & -82 & 20 & $62.12 / 6.77$ & 223 \\
\hline IOG & L & 19 & -44 & -72 & -4 & $59.84 / 6.67$ & 398 \\
\hline IOG & R & 19 & 48 & -66 & -10 & $48.84 / 6.13$ & 272 \\
\hline MOG & L & 19 & -32 & -82 & 20 & $39.46 / 5.60$ & 42 \\
\hline
\end{tabular}

Spatial coordinates, anatomical locations, and cluster size of the local maxima in the group analysis showing significant activations ( $p \leq 0.05$, corrected for multiple comparisons at the voxel level). IOG, Inferior occipital gyrus; L, left hemisphere; MOG, middle occipital gyrus; $\mathrm{PHC}$, parahippocampal cortex; R, right hemisphere.

the lateral retrosplenial cortices (Brodmann areas 23, 29, and 30; Fig. $3 a$, Table 1). In addition, we identified seven clusters in occipital cortex corresponding to Brodmann areas 17, 18, and 19; Table 1).

Using the conjunction analysis described in the previous section, we identified several additional clusters that were engaged by contextual mnemonic processes regardless of stimulus category or modality. The highest level of activity was observed in a large inferior frontal cluster, corresponding approximately to Broca's area (Table 2). In addition, we observed activity in the medial retrosplenial cortex (Brodmann area 30; Fig. 3b, Table 2), as well as the medial frontal and middle temporal cortex (Table 2).

The retrosplenial cortex has been implicated in both the perceptual analysis of scene layouts (Epstein, 2008) and the retrieval of associative contextual memories (Bar and Aminoff, 2003). Our results show that the lateral retrosplenial cortex is engaged during visual scene processing, whereas its medial portion is involved in contextual retrieval, suggesting the existence of functionally segregated subregions for contextual and visuospatial processing.

\section{Discussion}

Previous research has shown that the parahippocampal cortex is involved in visuospatial analysis of scenes (Epstein, 2008), as well
Table 2. Summary of fMRI findings for the conjunction analysis

\begin{tabular}{|c|c|c|c|c|c|c|c|}
\hline \multirow[b]{2}{*}{ Region } & \multirow[b]{2}{*}{ Hemisphere } & \multirow{2}{*}{$\begin{array}{l}\text { Brodmann } \\
\text { area }\end{array}$} & \multicolumn{3}{|c|}{$\begin{array}{l}\text { Activation maxima } \\
\text { MNI coordinates }\end{array}$} & \multirow{2}{*}{$\begin{array}{l}t \text {-values/ } \\
Z \text {-values of } \\
\text { maxima }\end{array}$} & \multirow{2}{*}{$\begin{array}{l}\text { Cluster } \\
\text { size (no. } \\
\text { of voxels) }\end{array}$} \\
\hline & & & $x$ & $y$ & $z$ & & \\
\hline IFG & L & $45 / 47$ & -38 & 30 & -12 & 11.32/Inf & 2740 \\
\hline $\begin{array}{l}\text { Retrosplenial } \\
\text { cortex }\end{array}$ & $L / R$ & 30 & -6 & -54 & 14 & 9.49/Inf & 219 \\
\hline PHC & L & $30 / 37$ & -30 & -32 & -20 & $8.46 / 7.26$ & 271 \\
\hline MedFG & $L / R$ & $9 / 10 / 32$ & -10 & 56 & 34 & $8.18 / 7.07$ & 270 \\
\hline MTG & $\mathrm{L}$ & 21 & -60 & -48 & 0 & $7.09 / 6.32$ & 351 \\
\hline
\end{tabular}

Spatial coordinates, anatomical locations, and cluster size of the local maxima in the group analysis showing significant activations ( $p \leq 0.05$, corrected for multiple comparisons at the voxel level). IFG, Inferior frontal gyrus; L, left hemisphere; MedFG, medial frontal gyrus; MTG, middle temporal gyrus; PHC, parahippocampal cortex; R, right hemisphere.

as contextual mnemonic processing (Aminoff et al., 2013). Until now, however, it has remained unclear whether these two processes are functionally and anatomically segregated within the parahippocampal cortex. We observed that, whereas the posterior parahippocampal cortex was strongly modulated bilaterally by the visual properties of the stimulus material, as evidenced by strong responses in the picture-scene conditions and no significant response in the word-object conditions, the left anterior parahippocampal cortex was active in all contextual judgment task conditions. This suggests a functional segregation, with posterior sections of the parahippocampal cortex supporting visuospatial processing of scenes and anterior sections of the left parahippocampal cortex involved in contextual associative processing. It is important to note that, whereas our study only examined retrieval-related activity for object-object and objectscene relationships, findings from prior studies suggest that the functions of the anterior parahippocampal cortex are not limited to these types of material, but should also be involved in the retrieval of associations involving other stimulus categories such as faces (Düzel et al., 2003; Kirwan and Stark, 2004).

A recent study by Aminoff and Tarr (2015) found activity within the posterior parahippocampal cortex when participants were shown previously viewed spatial configurations of meaningless shapes (regardless of their individual identities), whereas the anterior parahippocampal cortex was more active during the display of previously viewed meaningless shapes (regardless of their spatial configuration). Consistent with our results, these findings can also be interpreted as suggestive of anterior parahippocampal 
contextual processes and posterior parahippocampal visuospatial processes. Similarly, using an fMRI searchlight analysis, Linsley and McEvoy (2015) found that activity patterns in the anterior parahippocampal cortex were strongly modulated by the presence of objects in scenes, whereas those in the posterior parahippocampal gyrus were not, again suggesting that the anterior parahippocampal cortex is more concerned with contextual than visuospatial analysis of scene images.

Our proposal of a functional segregation along the parahippocampal longitudinal axis is also consistent with findings from a recent fMRI study (Baldassano et al., 2013), which found that anterior voxels in the parahippocampal cortex were more strongly functionally connected to parieto-medial temporal networks (i.e., regions supporting episodic, semantic, and spatial memories), whereas posterior voxels were more strongly functionally connected to occipital networks (i.e., regions concerned with visual processing). Together with the results of the present study, the findings of Baldassano et al. (2013) suggest that the functional topographic segregation of the parahippocampal cortex is underpinned by corresponding segregated connectivity patterns. More broadly, the findings are consistent with a model of parahippocampal function in which visual information is projected from occipital and temporal areas to the posterior parahippocampal cortex (Libby et al., 2012), where encoding of visuospatial information takes place, a process that is computationally more demanding for complex stimuli such as scenes than for simple ones such as objects (Chai et al., 2010). Conversely, we suggest that the anterior parahippocampal cortex encodes incoming information in an amodal format and acts as an interface with other memory regions, including the hippocampal, retrosplenial, and perirhinal memory systems (for an overview, see Ranganath and Ritchey, 2012).

Damage to the parahippocampal cortex can cause impairments in the identification of scenes, as well as deficits in forming new associative memories (Landis et al., 1986; Malkova and Mishkin, 2003). To date, however, human neuropsychological studies have not clearly differentiated between cases in which damage affected anterior or more posterior regions of the parahippocampal cortex (Bohbot et al., 1998, 2000). Based on our results, we would predict that focal lesions of the posterior parahippocampal cortex should impair performance selectively for conditions in which patients must encode or retrieve pictorial scenes because object- and word-processing pathways would remain intact and thus continue to mediate encoding processes for this type of stimulus material. In contrast, a focal lesion of the anterior parahippocampal cortex should lead to wide-ranging impairments in retrieving semantic associations because it would directly disrupt contextual associative memory processes for a wider range of stimulus categories. Future patient studies using high-resolution lesion symptom mapping should be able to test this prediction (Rorden et al., 2007).

Although our data suggest that the posterior parahippocampal cortex is involved in processing spatial features of visual scenes, it is still unclear how visuospatial properties are encoded at the neural level. Previous neuroimaging studies in humans and monkeys have shown that the parahippocampal cortex is robustly engaged in response to depictions of empty 3D spaces devoid of objects, scene layouts, or contextual associations, provided the stimulus contains sufficient high spatial frequency information (Rajimehr et al., 2011; Zeidmann et al., 2012). Further, a recent study by Nasr and colleagues (2014) showed that areas corresponding to the posterior PPA responded more strongly to simple rectilinear visual features, whereas those corresponding to the anterior PPA responded more strongly to complex visuospatial stimuli. However, it has not yet been established whether the parahippocampal cortex responds selectively to different visuospatial features such as textures, colors, and boundaries (Epstein, 2008). It also remains unclear whether the manner in which the anterior parahippocampal cortex encodes and retrieves associative memories differs from that of other regions known to be involved in these processes, such as the hippocampal and retrosplenial regions (Mayes et al., 2007; Vann et al., 2009). Moreover, it is conceivable that different categories of contextual associations (e.g., spatial, temporal, affective, etc.) cluster in different subregions within the anterior parahippocampal cortex. Highresolution $\mathrm{fMRI}$ and analysis techniques such as adaptation $\mathrm{fMRI}$ (Krekelberg et al., 2006) or multivoxel pattern classification (Norman et al., 2006) could be used to determine any such finegrained functional topography within the parahippocampal cortex.

\section{References}

Aminoff EM, Tarr MJ (2015) Associative processing is inherent in scene perception. PLoS One 10:e0128840. CrossRef Medline

Aminoff EM, Kveraga K, Bar M (2013) The role of the parahippocampal cortex in cognition. Trends Cogn Sci 17:379-390. CrossRef Medline

Baldassano C, Beck DM, Fei-Fei L (2013) Differential connectivity within the parahippocampal place area. Neuroimage 75:228-237. CrossRef Medline

Bar M, Aminoff E (2003) Cortical analysis of visual context. Neuron 38: 347-358. CrossRef Medline

Bar M, Aminoff E, Ishai A (2008) Famous faces activate contextual associations in the parahippocampal cortex. Cereb Cortex 18:1233-1238. CrossRef Medline

Bar M, Aminoff E, Schacter DL (2008) Scenes unseen: the parahippocampal cortex intrinsically subserves contextual associations, not scenes or places per se. J Neurosci 28:8539-8544. CrossRef Medline

Baumann O, Chan E, Mattingley JB (2010) Dissociable neural circuits for encoding and retrieval of object locations during active navigation in humans. Neuroimage 49:2816-2825. CrossRef Medline

Bohbot VD, Allen JJ, Nadel L (2000) Memory deficits characterized by patterns of lesions to the hippocampus and parahippocampal cortex. Ann N Y Acad Sci 911:355-368. Medline

Bohbot VD, Kalina M, Stepankova K, Spackova N, Petrides M, Nadel L (1998) Spatial memory deficits in patients with lesions to the right hippocampus and to the right parahippocampal cortex. Neuropsychologia 36:1217-1238. CrossRef Medline

Chai XJ, Ofen N, Jacobs LF, Gabrieli JD (2010) Scene complexity: influence on perception, memory, and development in the medial temporal lobe. Front Hum Neurosci 4:21. Medline

Düzel E, Habib R, Rotte M, Guderian S, Tulving E, Heinze HJ (2003) Human hippocampal and parahippocampal activity during visual associative recognition memory for spatial and nonspatial stimulus configurations. J Neurosci 23:9439-9444. Medline

Epstein RA (2005) The cortical basis of visual scene processing. Vis Cogn 12:954-978. CrossRef

Epstein RA (2008) Parahippocampal and retrosplenial contributions to human spatial navigation. Trends Cogn Sci 12:388-396. CrossRef Medline

Epstein R, Kanwisher N (1998) A cortical representation of the local visual environment. Nature 392:598-601. CrossRef Medline

Epstein RA, Ward EJ (2010) How reliable are visual context effects in the parahippocampal place area? Cereb Cortex 20:294-303. CrossRef Medline

Epstein R, Harris A, Stanley D, Kanwisher N (1999) The parahippocampal place area: recognition, navigation, or encoding? Neuron 23:115-125. CrossRef Medline

Epstein R, Graham KS, Downing PE (2003) Viewpoint-specific scene representations in human parahippocampal cortex. Neuron 37:865-876. CrossRef Medline

Janzen G, van Turennout M (2004) Selective neural representation of objects relevant for navigation. Nat Neurosci 7:673-677. CrossRef Medline Kirwan CB, Stark CE (2004) Medial temporal lobe activation during encod- 
ing and retrieval of novel face-name pairs. Hippocampus 14:919-930. CrossRef Medline

Krekelberg B, Boynton GM, van Wezel RJ (2006) Adaptation: from single cells to BOLD signals. Trends Neurosci 29:250-256. CrossRef Medline

Landis T, Cummings JL, Benson DF, Palmer EP (1986) Loss of topographic familiarity: an environmental agnosia. Arch Neurol 43:132-136. CrossRef Medline

Libby LA, Ekstrom AD, Ragland JD, Ranganath C (2012) Differential connectivity of perirhinal and parahippocampal cortices within human hippocampal subregions revealed by high-resolution functional imaging. J Neurosci 32:6550-6560. CrossRef Medline

Linsley D, MacEvoy SP (2015) Encoding-stage crosstalk between objectand spatial property-based scene processing pathways. Cereb Cortex 25: 2267-2281. CrossRef Medline

Maldjian JA, Laurienti PJ, Kraft RA, Burdette JH (2003) An automated method for neuroanatomic and cytoarchitectonic atlas-based interrogation of fMRI data sets. Neuroimage 19:1233-1239. CrossRef Medline

Malkova L, Mishkin M (2003) One-trial memory for object-place associations after separate lesions of hippocampus and posterior parahippocampal region in the monkey. J Neurosci 23:1956-1965. Medline

Mayes A, Montaldi D, Migo E (2007) Associative memory and the medial temporal lobes. Trends Cogn Sci 11:126-135. CrossRef Medline

Nasr S, Echavarria CE, Tootell RB (2014) Thinking outside the box: rectilinear shapes selectively activate scene-selective cortex. J Neurosci 34: 6721-6735. CrossRef Medline

Nichols T, Brett M, Andersson J, Wager T, Poline JB (2005) Valid conjunction inference with the minimum statistic. Neuroimage 25:653-660. CrossRef Medline

Norman KA, Polyn SM, Detre GJ, Haxby JV (2006) Beyond mind-reading: multi-voxel pattern analysis of fMRI data. Trends Cogn Sci 10:424-430. CrossRef Medline

Park S, Chun MM (2009) Different roles of the parahippocampal place area
(PPA) and retrosplenial cortex (RSC) in panoramic scene perception. Neuroimage 47:1747-1756. CrossRef Medline

Rajimehr R, Devaney KJ, Bilenko NY, Young JC, Tootell RB (2011) The "parahippocampal place area" responds preferentially to high spatial frequencies in humans and monkeys. PLoS Biol 9:e1000608. CrossRef Medline

Ranganath C, Ritchey M (2012) Two cortical systems for memory-guided behaviour. Nat Rev Neurosci 13:713-726. CrossRef Medline

Rorden C, Karnath HO, Bonilha L (2007) Improving lesion-symptom mapping. J Cogn Neurosci 19:1081-1088. CrossRef Medline

Tzourio-Mazoyer N, Landeau B, Papathanassiou D, Crivello F, Etard O, Delcroix N, Mazoyer B, Joliot M (2002) Automated anatomical labeling of activations in SPM using a macroscopic anatomical parcellation of the MNI MRI single-subject brain. Neuroimage 15:273-289. CrossRef Medline

Vann SD, Aggleton JP, Maguire EA (2009) What does the retrosplenial cortex do? Nat Rev Neurosci 10:792-802. CrossRef Medline

Wegman J, Janzen G (2011) Neural encoding of objects relevant for navigation and resting state correlations with navigational ability. J Cogn Neurosci 23:3841-3854. CrossRef Medline

Weiskopf N, Hutton C, Josephs O, Deichmann R (2006) Optimal EPI parameters for reduction of susceptibility-induced BOLD sensitivity losses: a wholebrain analysis at $3 \mathrm{~T}$ and $1.5 \mathrm{~T}$. Neuroimage 33:493-504. CrossRef Medline

Zaitsev M, Steinhoff S, Shah NJ (2003) Error reduction and parameter optimization of the TAPIR method for fast T1 mapping. Magn Reson Med 49:1121-1132. CrossRef Medline

Zeidman P, Mullally SL, Schwarzkopf DS, Maguire EA (2012) Exploring the parahippocampal cortex response to high and low spatial frequency spaces. Neuroreport 23:503-507. CrossRef Medline

Zeng H, Constable RT (2002) Image distortion correction in EPI: comparison of field mapping with point spread function mapping. Magn Reson Med 48:137-146. CrossRef Medline 